\title{
A new algorithm for improving fetal weight estimation from ultrasound data at term
}

\author{
W. Siggelkow $\cdot$ M. Schmidt $\cdot$ C. Skala $\cdot$ D. Boehm $\cdot$ \\ S. von Forstner $\cdot$ H. Koelbl $\cdot$ A. Tresch
}

Received: 24 August 2009/Accepted: 2 February 2010/Published online: 20 February 2010

(C) The Author(s) 2010. This article is published with open access at Springerlink.com

\begin{abstract}
Objective The purpose of this retrospective study was to find a method of improving the accuracy of fetal birth weight estimation on the basis of traditional ultrasonographic measurements of the head, thorax, and femur at term. In this context, we analyzed a novel regression method comparing to existing algorithms.

Methods The delivery records of two hospitals were searched for women who delivered macrosomic infants, and the patients' medical records were retrospectively reviewed in order to derive clinical and ultrasonographic data at term. A total of 223 patients with macrosomic infants (birth weight $>4,000 \mathrm{~g}$ ) were identified. These patients were complemented by data for 212 women who had ultrasound fetal assessments of less than 4,000 g. We used the method of isotonic regression to construct a birth weight prediction function that increases monotonically with each of the input variables and which minimizes the empirical quadratic loss.

Results A suspicion of macrosomia was based on a history of macrosomia, fundal height, and sonographic weight estimation $>4,000 \mathrm{~g}$. The mean period between ultrasound weight estimation and delivery was 7.2 days. The ability of
\end{abstract}

W. Siggelkow $\cdot$ M. Schmidt $\cdot$ C. Skala $\cdot$ D. Boehm ·

S. von Forstner $\cdot$ H. Koelbl

Department of Obstetrics and Gynecology,

Johannes Gutenberg University, Mainz, Germany

A. Tresch

Gene Center, Department of Chemistry and Biochemistry,

LMU, Muenchen, Germany

W. Siggelkow ( $\square)$

Department of Obstetrics and Gynecology, University of Mainz,

Langenbeck-Strasse 1, 55101 Mainz, Germany

e-mail: siggelko@uni-mainz.de the biometric algorithms developed to predict fetal weight at term ranged between a mean absolute error of 312 and $344 \mathrm{~g}$, given a confidence interval of $95 \%$. We demonstrate that predictions of birth weight on the basis of ultrasound data can be improved significantly, if an isotonic regression model is used instead of a linear regression model.

Conclusions This study demonstrates that ultrasound detection of macrosomia can be improved using the isotonic regression method.

Keywords Fetal weight estimation - Fetal macrosomia . Birth weight algorithms · Isotonic regression

\section{Introduction}

The accurate prediction of the estimated birth weight at term has a major impact on the appropriate obstetric management for the fetus in particular in the cases of suspected macrosomia. Macrosomia, defined as a birth weight exceeding $4,000 \mathrm{~g}$, accounts for almost $10 \%$ of all births [1-3]. Delivery of a macrosomic fetus may increase both maternal and perinatal morbidity. Macrosomia is associated with shoulder dystocia and birth asphyxia and trauma, as well as increases in the cesarean delivery rate and postpartum hemorrhage $[4,5]$. New data for reliable fetal weight estimation by measurement of fetal soft tissue were recently presented [6-8]. Unfortunately, ultrasound assessments of estimated fetal weight are particularly inaccurate among neonates with birth weights greater than $4,000 \mathrm{~g}$ [9]. The ability of ultrasound fetal biometry to estimate fetal weight in comparison with clinical methods is still a matter of concern in the presence of macrosomia $[10,11]$. The precision of ultrasound birth weight estimation has been challenged in numerous studies, which have 
concluded that sonography may be no more accurate in predicting birth weight than clinical methods. To date, a gold standard for predicting term birth weight has not yet been established [12-14].

It is assumed that ultrasound measurement of the multiple linear and planar dimensions of the fetus provides sufficient parametric information to allow for the accurate algorithmic reconstruction of the three-dimensional fetal volume of varying tissue density. In accordance with these assumptions, much effort has been expended on generating best-fit fetal biometric algorithms capable of providing birth weight predictions on the basis of obstetric ultrasound measurements [9].

The goal of the present study was to improve the accuracy of fetal weight estimation at term on the basis of traditional linear measurements of the head, thoracic diameter, and femur at term. The current approach is to match a linear model to the ultrasound data or some quantities derived from it. The birth weight is essentially a three-dimensional quantity whose dependence on the fetal weight is presumably non-linear. In the present investigation, we used the method of isotonic regression to construct a birth weight prediction function that increases monotonically with each of the input variables and which minimizes the empirical quadratic loss.

\section{Materials and methods}

\section{Data preparation}

The delivery records of the Department of Obstetrics and Gynecology at the University of Mainz, Germany, and of St. Joseph Hospital in Bremen, Germany, were searched for women who had delivered macrosomic infants. Their medical records were retrospectively reviewed to obtain clinical and ultrasonographic data at term. Patients were included in this study if the following criteria were satisfied:

- Single gestation

- Accurate assessment of gestational age on the basis of a reliable date for the last menstrual period or ultrasonogram 14 weeks previously

- Ultrasound assessment of the biparietal diameter, occipitofrontal diameter, abdominal diameter, and femur length

- A maximum time between ultrasound and delivery of 10 days

Most patients had the ultrasound examinations performed within 1 week of delivery. The patients were not in labor and had intact membranes at the time of the ultrasound examinations. The retrieved medical records were gained in a time period of 10 months. The patient's characteristics are summarized in Table 1. All records of macrosomic and normosomic infants were retrieved consecutively. All of the ultrasound examinations were carried out by experienced residents trained in ultrasound. A total of 223 patients with macrosomic infants (estimated weight $>4,000 \mathrm{~g}$ ) were identified, representing $8.2 \%$ of the general obstetric population in these departments. These patients were complemented by 212 women who had ultrasound measurements resulting in estimated birth of less than 4,000 g. In comparison to the overall population birth weight distribution, the birth weight distribution of the samples has been deliberately skewed toward larger weights. This was done in order to obtain enough data in the region of interest, allowing for increased prediction accuracy at the right tail of the distribution. All fetal sonographic weight estimations were carried out using ATL Ultramark 9 HDI ultrasound systems (Philips Medical Systems Ltd., Hamburg, Germany).

The anatomical landmarks of the ultrasound examination were the occipitofrontal and the biparietal diameter at its maximum. The thoracic diameter was measured at the lower thoracic aperture, giving a four-chamber view of the heart.

The chart data that were extracted for each patient included maternal ethnic background, age, gravidity, parity, height, and weight development during pregnancy, final pregnancy weight, presence of gestational diabetes, complications of pregnancy, smoking history, and delivery data, including delivery weight. Birth weight was predicted from each sonogram using the gestation-adjusted projection method described by Hadlock et al. with the median fetal weight for gestational age $[15,16]$.

\section{Statistical analysis}

Complete data were derived from 286 patients, for whom three ultrasonic parameters were measured at term: biparietal distance (BPD), thoracic diameter (THD), and femur length (FL). Several regression methods used to predict birth weight (BW) from these data; for a complete list, see Table 1. Accuracy was assessed in terms of the mean absolute deviation from the true birth weight. We used a 10-fold cross-validation method in order to obtain

Table 1 Characteristics of the patients

\begin{tabular}{lll}
\hline Characteristics & Mean & Range \\
\hline Maternal age (years) & 29 & $16-43$ \\
Parity & 1 & $1-8$ \\
Gestational age at delivery (weeks) & 38.5 & $37.4-41.6$ \\
Birth weight (g; ultrasound estimate) & 3,561 & $1,895-5,014$ \\
Absolute birth weight (g) & 3,798 & $2,170-5,180$ \\
\hline
\end{tabular}


a reliable estimate of the prediction accuracy of each algorithm.

The only regression models used for birth weight prediction to date have been linear models [17-20]. We included many of these in our analysis, although they had to be slightly adapted to our situation; abdominal circumference-a parameter widely used in ultrasound diagnosis-was not measured in this study. We used the thoracic diameter as a proxy of abdominal circumference if necessary, assuming that both quantities resemble each other. Note that we merely extracted the structure of the regression formula from the literature, we did not adopt the coefficients. Those were estimated from our data to make the comparison fair. It is highly unlikely that replacement of abdominal circumference by THD impairs the prediction accuracy of, e.g., Hadlock's formulas significantly, but we admit that we cannot definitely rule out this possibility.

The key observation we made is that the most simple linear model essentially produces the formula

$\mathrm{BW}_{\text {linear }}(\mathrm{BPD}, \mathrm{THD}, \mathrm{FL})=\mathrm{c} \cdot(\mathrm{BPD}+\mathrm{THD}+\mathrm{FL})+\mathrm{c}_{0}$

for a positive constant $c=2.57 \mathrm{~g} / \mathrm{cm}$ and an intercept $c_{0}=-3,330 \mathrm{~g}$ (i.e., the regression coefficients of BPD, THD, and FL were almost identical). (Removed: As a linear function of the one-dimensional variables BPD, THD, and FL, the linear model produces quantity which is a reliable estimate of the fetal length (up to linear rescaling)). However, the birth weight is essentially a threedimensional quantity whose dependence on the fetal length is presumably non-linear. Therefore, in a second step, we take a nonparametric shape-constrained regression approach. Instead of using the above linear regression as a predictor of the fetal length, we find a best-fitting birth weight prediction function that increases monotonically with the fetal length (this is known as an isotonic regression function) [18]. Just as for linear regression, goodness of fit is measured in terms of the mean quadratic prediction error on the training data. As a nonparametric method, isotonic regression finds a piecewise constant; monotonically increasing function which cannot be given as a closed-form, smooth prediction formula.

\section{Results}

The study population consisted of 435 patients undergoing ultrasound examinations on entering a department of obstetrics and gynecology for delivery at term. A total of 223 of the newborns $(51.5 \%)$ had birth weights $>4,000 \mathrm{~g}$. The number of cases of gestational diabetes in macrosomic infants was 19 (8.6\%), compared with 21 (10.1\%) in normosomic infants, yielding no statistical difference in the incidence of diabetes between the normosomic and macrosomic infant populations. The indication for ultrasound was weight estimation at term in normosomic and macrosomic infants before vaginal delivery. The suspicion of macrosomia was based on a history of macrosomia, fundal height, and a sonographic weight estimation $>4,000 \mathrm{~g}$.

The mean gestational age at delivery was 38.5 weeks, and the mean time between the ultrasound weight estimations and delivery was 7.2 days. The patients' characteristics are shown in Table 2.

With regard to cross-validation, the overall ability of the ultrasound algorithms used to predict fetal weight at term error ranged between 312 and 354 g, given a confidence interval of $95 \%$.

The results show that all of the methods performed comparably, although isotonic regression was significantly better than the others (Table 2). It turned out that linear methods performed better with square-transformed birth weight data. Being a nonparametric method it is no surprise that the performance of the isotonic regression is virtually unaffected by (monotonic) data transformation. The mean absolute deviation from the true birth weight ranged from about 310-340 g. The distribution of the deviations of the predicted weights from the true birth weights is shown in Fig. 1. The ROC of all methods are pictured in Fig. 2. Each regression method can be turned into a classifier for

Table 2 Performance of six regression methods on untransformed data

\begin{tabular}{lll}
\hline Method & Regression variables & $\begin{array}{l}\text { Accuracy (cross-validation error), } \\
\text { 95\% confidence interval (mean values) }\end{array}$ \\
\hline Isotonic regression & $\mathrm{BPD}, \mathrm{THD}, \mathrm{FL}$ & $312(307-318)$ \\
Hadlock (16) & $\mathrm{BPD}, \mathrm{BPD}^{2}, \mathrm{THD}, \mathrm{THD}^{2}, \mathrm{BPD} \bullet \mathrm{THD}$ & $331(329-334)$ \\
Linear & $\mathrm{BPD}, \mathrm{THD}, \mathrm{FL}$ & $338(336-341)$ \\
Warda (30) & $\mathrm{BPD}, \mathrm{THD}{ }^{2}, \mathrm{FL}, \mathrm{BPD} \bullet \mathrm{THD}^{2}, \mathrm{THD} \bullet \mathrm{FL}$ & $340(337-343)$ \\
Shinozuka (29) & $\mathrm{BPD}^{3}, \mathrm{THD}^{2} \bullet \mathrm{FL}$ & $344(342-346)$ \\
Warsof (31) & $\mathrm{FL}, \mathrm{THD}^{2}, \mathrm{THD} \bullet \mathrm{FL}$ & $354(351-358)$
\end{tabular}

For each method, the quantities that actually enter the regression function are listed (e.g., Shinozuka uses only two input variables, namely BPD and the product of THD and FL). The accuracy is given as the mean absolute deviation from the true weight. Using cross-validation, 95\% confidence intervals have been obtained for this deviation 


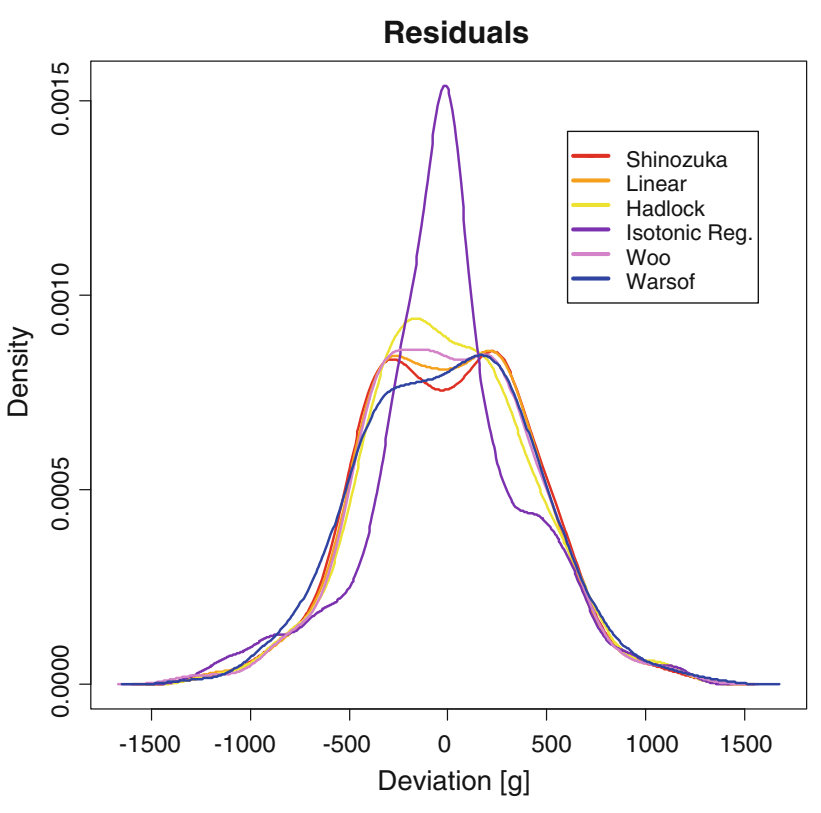

Fig. 1 Distribution of the regression residuals for the six regression methods shown in Fig. 1, using untransformed data

macrosomia by deciding for macrosomia $(>4,000 \mathrm{~g})$ if the predicted weight exceeds some defined threshold value. The receiver operating characteristics (ROC) of all classifiers obtained that way are given in Fig. 2. All results of this article, including an $\mathrm{R}$ script reproducing the results, plus supplementary information can be accessed online at http://www.lmb.uni-muenchen.de/tresch/supplements_ birthweights.html.

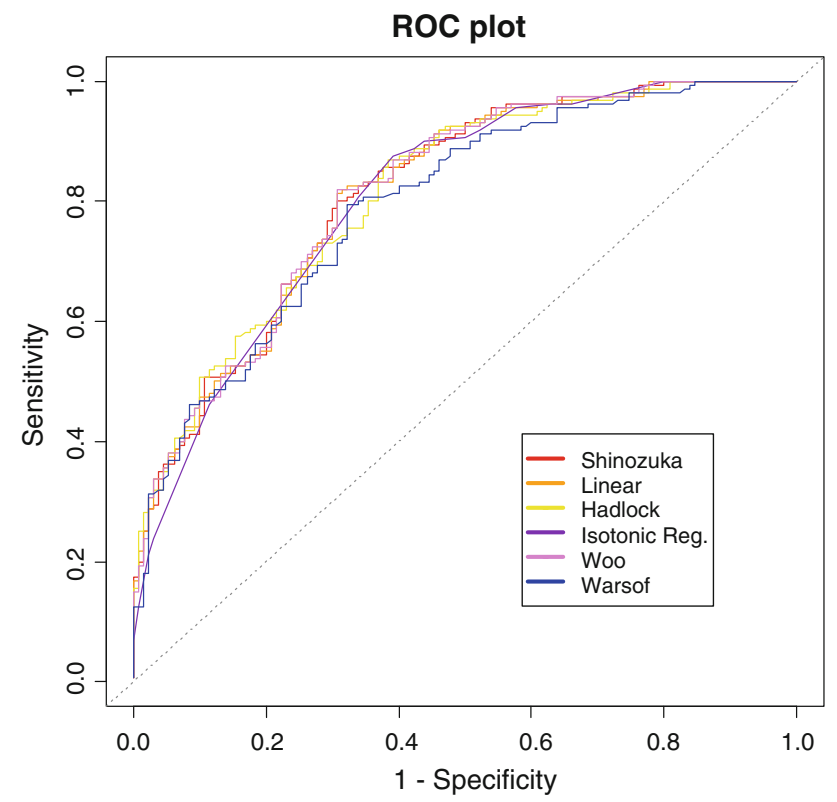

Fig. 2 Six regression methods and their performance as predictors of macrosomia. The receiver operating characteristics (ROC) of all of the methods appear very similar-i.e., all of the methods perform equally well

\section{Discussion}

The established complications of macrosomia indicate that there is a need to identify large fetuses prior to labor $[5,9,10$, 21]. However, the results with ultrasound estimation of fetal weight have been disappointing, in view of the low sensitivity and specificity values achieved in macrosomic fetuses in particular $[13,22]$. The accuracy of fetal weight estimation using the formula presented by Hadlock et al. has been reported to be markedly decreased in fetuses weighing $>4,000 \mathrm{~g}$. Hadlock et al. quite reasonably calculated their equation in order to maximize the accuracy in estimating fetal weight for the largest number of fetuses, rather than for outliers such as macrosomic fetuses $[15,16]$. In earlier studies, the possibility has been discussed that different groups of patients may require different equations for better estimation. Equations that are specifically tailored to group characteristics that include maternal height and weight yield a sensitivity for predicting birth weight $>4,000 \mathrm{~g}$ and birth weight $>4,500 \mathrm{~g}$ that is significantly greater than that obtained with the Hadlock et al. equation $[9,16]$. Additional assumptions have been that the ultrasound measurement of multiple linear or planar dimensions of the fetus provides sufficient parametric information to allow accurate algorithmic reconstruction of the three-dimensional fetal volume of varying tissue density.

In accordance with these beliefs, a great deal of effort has been put into generating best-fit fetal biometric algorithms capable of making birth weight predictions on the basis of obstetric ultrasound measurements [16, 23-30]. Nahum et al. assessed 20 standard ultrasonic fetal biometric algorithms for their accuracy in predicting term fetal weight. These algorithms included the most commonly used ultrasonographic fetal biometric equations, which are based on various combinations of the fetal measurements as well as maternal and pregnancy-specific characteristics. The accuracy of fetal weight predictions was quantified by calculating the correlation with actual birth weight, the mean squared error, the mean absolute percentage error, and the percentage of birth weights that were predicted accurately to within $\pm 10 \%$ and to within $\pm 15 \%$ of the actual birth weight. With regard to their ability to predict fetal weight to within $\pm 10 \%$ of actual birth weight, the 20 ultrasound equations ranged in accuracy from a minimum of $23 \%$ based on $\mathrm{AC}$ and FL to a maximum of $74 \%$ based on $\mathrm{AC}$ alone. The maternal characteristics equation was the most accurate of all approaches for predicting fetal macrosomia, with a sensitivity of $55 \%$, a specificity of $90 \%$, and a positive predictive value of $55 \%$ for detecting fetal weight $>4,000$ [9].

In earlier studies, thoracic diameter has been a single predictor of birth weight [31, 32]. It should be noted that many studies fail to assess the prediction accuracy of their algorithms reliably using independent test data, cross- 
validation, or bootstrap methods. The results published by Sokol et al. showed that head circumference and maternal data such as height and weight can play an important role in improving the sensitivity for the presence of macrosomia [13].

The aim of the present investigation was to derive an algorithm for a more accurate ultrasound estimation of birth weight, to enable obstetricians to offer better counseling to parents regarding the risks associated with vaginal delivery of a macrosomic fetus. With a mean absolute deviation of about $310 \mathrm{~g}$ from the true birth weight, the overall performance of the algorithm developed was moderate, but significantly better than that of existing methods. This may be due to the fact that other variables such as fetal sex and the mother's smoking habits and ethnic background, which may have independent effects on birth weight, could not be taken into account because of the retrospective nature of this study.

It has been demonstrated that predictions of birth weight on the basis of ultrasound data can be improved slightly, but significantly, if an isotonic regression model is used instead of a linear regression model. The accuracy of sonographic birth weight estimation is known to vary with gestational age. The gestational period between 34.0 and 36.9 weeks has been regarded as a better time for obtaining fetal measurements to predict subsequent birth weight, rather than later in gestation $[3,9,12]$. We studied macrosomic fetuses near term in order to define ultrasound-based equations, since most of our patients enter the delivery unit immediately before labor or at the start of labor, so that there is no opportunity for more accurate ultrasound assessment at earlier stages of pregnancy. The patients were screened a few days before delivery and before labor.

In conclusion, this study has some limitations. Our results were derived from retrospective data and demonstrate that ultrasound detection of macrosomia can be improved using techniques such as isotonic regression. The clinical value of this investigation can be improved with a prospective setting. In addition, equations need to be optimized for detecting macrosomia by stressing fit in the range of interest; for example, in decision making concerning the route of delivery [13].

\section{Conflict of interest statement None.}

Open Access This article is distributed under the terms of the Creative Commons Attribution Noncommercial License which permits any noncommercial use, distribution, and reproduction in any medium, provided the original author(s) and source are credited.

\section{References}

1. Chauhan SP et al (2000) Antepartum detection of macrosomic fetus: clinical versus sonographic, including soft-tissue measurements. Obstet Gynecol 95(5):639-642
2. Chauhan SP et al (1993) Intrapartum prediction of birth weight: clinical versus sonographic estimation based on femur length alone. Obstet Gynecol 81(5 (Pt 1)):695-697

3. Nahum GG, Stanislaw H (2000) Prediction of birth weight by ultrasound in the third trimester. Obstet Gynecol 96(2):319-320

4. Shepard MJ, Hellenbrand KG, Bracken MB (1986) Proportional weight gain and complications of pregnancy, labor, and delivery in healthy women of normal prepregnant stature. Am J Obstet Gynecol 155(5):947-954

5. Combs CA et al (2000) Sonographic EFW and macrosomia: is there an optimum formula to predict diabetic fetal macrosomia? J Matern Fetal Med 9(1):55-61

6. Larciprete G, Di Pierro G, Barbati G, Deaibess T, Jarvis S, Valensise H, Romanini ME, Gioia S, Arduini D (2008) Could birthweight prediction models be improved by adding fetal subcutaneous tissue thickness? J Obstet Gynecol Res 34:18-26

7. Larciprete G, Valensise H, Barbati G, Di Pierro G, Jarvis S, Deaibess T, Gioia S, Giacomello F, Cirese E, Arduini D (2007) Ultrasound-determined fetal subcutaneous tissue thickness for a birthweight prediction model. J Obstet Gynaecol Res 33:635-640

8. Scioscia M, Scioscia F, Vimercati A, Caradonna F, Nardelli C, Pinto LR, Selvaggi LE (2008) Estimation of the fetal weight by measurement of fetal thigh soft-tissue thickness in the late third trimester. Ultrsound Obstet Gynecol 31:314-320

9. Nahum GG, Stanislaw H (2003) Ultrasonographic prediction of term birth weight: how accurate is it? Am J Obstet Gynecol 188(2):566-574

10. Chauhan SP et al (2000) Sonographic measurements of fetal parts to predict pulmonary maturity among twins and singletons. J Miss State Med Assoc 41(3):516-520

11. Hendrix NW, Grady CS, Chauhan SP (2000) Clinical vs. sonographic estimate of birth weight in term parturients. A randomized clinical trial. J Reprod Med 45(4):317-322

12. Pressman EK et al (2000) Prediction of birth weight by ultrasound in the third trimester. Obstet Gynecol 95(4):502-506

13. Sokol RJ et al (2000) Correctly identifying the macrosomic fetus: improving ultrasonography-based prediction. Am J Obstet Gynecol 182(6):1489-1495

14. Weiner $\mathrm{Z}$ et al (2002) Clinical and ultrasonographic weight estimation in large for gestational age fetus. Eur J Obstet Gynecol Reprod Biol 105(1):20-24

15. Hadlock FP et al (1984) Sonographic estimation of fetal weight. The value of femur length in addition to head and abdomen measurements. Radiology 150(2):535-540

16. Hadlock FP et al (1985) Estimation of fetal weight with the use of head, body, and femur measurements - a prospective study. Am J Obstet Gynecol 151(3):333-337

17. Barlow RE, Bartholomew DJ, Bremner JM, Brunk HD (1972) Statistical inference under order restrictions; the theory and application of isotonic regression. Wiley, New York

18. Kruskal JB (1964) Nonmetric multidimensional scaling: a numerical method. Psychometrica 29(2):115-129

19. Barlow RE et al (1972) In: Barlow RE (ed) Statistical inference under order restrictions. The theory and application of isotonic regression. Wiley, New York

20. Johnstone IM, Silvermann BW (2005) Ebayes Thresh: R programs for empirical Bayes thresholding. J Statist Softw 12(8):1-38

21. Pates JA, McIntire DD, Casey BM, Leveno KJ (2008) Predicting macrosomia. J Ultrasound Med 27:39-43

22. Jazayeri A et al (1999) Macrosomia prediction using ultrasound fetal abdominal circumference of 35 centimeters or more. Obstet Gynecol 93(4):523-526

23. Henrichs C et al (2003) Detecting fetal macrosomia with abdominal circumference alone. J Reprod Med 48(5):339-342 
24. Higginbottom $\mathbf{J}$ et al (1975) Estimation of fetal weight from ultrasonic measurement of trunk circumference. $\mathrm{Br} \mathrm{J}$ Obstet Gynaecol 82(9):698-701

25. Hsieh FJ et al (1987) Computer-assisted analysis for prediction of fetal weight by ultrasound-comparison of biparietal diameter (BPD), abdominal circumference (AC) and femur length (FL). Taiwan Yi Xue Hui Za Zhi 86(9):957-964

26. Humphries J et al (2002) Sonographic estimate of birth weight: relative accuracy of sonographers versus maternal-fetal medicine specialists. J Matern Fetal Neonatal Med 11(2):108-112

27. Shepard MJ et al (1996) Maternal body mass, proportional weight gain, and fetal growth in parous women. Paediatr Perinat Epidemiol 10(2):207-219

28. Shinozuka N et al (1987) Formulas for fetal weight estimation by ultrasound measurements based on neonatal specific gravities and volumes. Am J Obstet Gynecol 157(5):1140-1145

29. Warda A et al (1986) Evaluation of fetal thigh circumference measurements: a comparative ultrasound and anatomical study. J Clin Ultrasound 14(2):99-103
30. Warsof SL et al (1977) The estimation of fetal weight by computer-assisted analysis. Am J Obstet Gynecol 128(8):881-892

31. Gerhard IVB, Runnebaum B, Klinga K, Haller U, Kubli F (1987) Weight percentile at birth. Prediction by endocrinological and sonographic measurements. Percentile at birth. II. Prediction by endocrinological and sonographic measurements. Eur J Obstet Gynecol Reprod Biol 26(4):313

32. Göttlicher SMJ (1988) Is a quantitatively exact prediction of expected birth weight possible using the sonographically determined parameters of biparietal diameter, thoracic diameter and length of the humerus? A prospective study of 285 pregnant patients birth weight possible using the sonographically determined parameters of biparietal diameter, thoracic diameter and length of the humerus? A prospective study of 285 pregnant patients. Zentralbl Gynakol 110(19):1206-1212 